\title{
THE DARBOUX POINT AND THE CONJUGATE POINT ON OPTIMAL DEORBIT FOR REENTRY TRAJECTORIES †
}

\author{
NGUYEN X. Vinh and Jennil: R. JOHANneslen \\ Department of Aerospace Enginecring, University of Michigan, Ann Arbor, \\ MI 48109-2140. U.S.A
}

(Received 28 Mardh 1985)

\begin{abstract}
The concept of the Darboux point at which an extremal loses its global optimality is extended to the case of discontinuous control. Using Contensou 's domain of maneuverability, the condition for optimal switching at a corner is derived and the optimality of the trajectory in the neighborhood of a Darboux point is analyzed. The theory is applied to the problems of minimum-fuel planar and noncoplanar deorbit from elliptical orbits for atmospheric entry at a prescribed angle. In each case, the global optimal trajectory is assessed and it is found that in these nonlinear problems the Darboux point and the conjugate point are distinct. The global optimality is always lost before local optimality.
\end{abstract}

\section{INTRODUCTION}

In optimal control the appearance of a conjugate point on an extremal indicates the loss of local optimality. It has been noted in several papers[1,2] that in some cases an extremal loses global optimality at a particular point. necessarily at or before the conjugate point. This point is called the Darboux point in recognition of Darboux's earlier discussion of this phenomenon when he studied the geodesies on a surface[3]. We shall adopt the following definition given in $|4|$

Definition 1. Let $y^{*}(t), t \in\left[t_{t}, t_{f}^{*}\right]$ be a trajectory which satisfies the maximum principle. A point $t_{t} \in\left[t_{t}\right.$, $\left.t_{i}^{*}\right)$ is called a Darboux point if: (i) for all $t_{2} \in\left(t_{D}, t_{i}^{*}\right)$, there does not exist an admissible trajectory $y(t), t \in \mid t_{2}$, $t_{f}$, with $y\left(t_{2}\right)=y^{*}\left(t_{2}\right)$, giving a smaller value to the cost functional between $t_{2}$ and $t_{t}$ than $y^{*}(t)$ between $t_{2}$ and $t_{f}^{*}$, and if (ii) for all $t_{1} \in\left[t_{i}, t_{0}\right)$, there exists an admissible trajectory $\hat{v}(t), t \in\left[t_{1}, \hat{t}_{j}\right]$, with $\hat{y}\left(t_{1}\right)=y^{*}\left(t_{1}\right)$, giving a smaller value to the cost functional between $t_{1}$ and $\hat{t}_{f}$ than $y^{*}(t)$ between $t_{1}$ and $t_{f}^{*}$.

This definition is illustrated in Fig. 1. From the definition it is easy to prove the existence of the Darboux point when there exist $t_{1}$ and $t_{2}, t_{0} \leq t_{1}<t_{2}<t_{i}^{*}$, such that $y^{*}$ is globally optimal on $\left[t_{2}, t_{f}^{*}\right]$ and not globally optimal on $\left[t_{1}, t_{f}^{*}\right]$. Then there certainly exists a Darboux point $t_{l}$ on $\left(t_{1}, t_{2}\right)$.

A classification of the Darboux point and the optimality of the trajectory $y^{*}$ in its neighborhood have been given in [4] for the class of problems where the control and all the functions involved in the definition of the problem are continuous. So far there have been no applications to a realistic and physical problem where the control can be discontinuous. In this paper, we shall use Contensou's domain of maneuverability[5] to investigate the optimality of the solution in the neighborhood of a Darboux point for the case where the extremal may include a corner.

$\div$ Paper presented at the 35 th Congress of the International Astronautical Federation, 8-13 October 1984
As applications of the theory, we consider the problems of optimal planar and noncoplanar impulsive deorbit of a space vehicle for entering the atmosphere, at distance

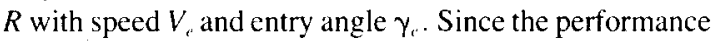
for subsequent atmospheric flight, either in the case of effective entry for landing (such as in the case of a shuttle), or atmospheric passage for aeroassisted maneuver (such as in the case of an orbital transfer vehicle), depends on the atmospheric entry condition, it is assumed that the deorbit must satisfy a certain constraint

$$
F\left(R, V_{i}, \gamma_{c}\right)=0
$$

This condition constitutes the terminal manifold in the phase space. In each case considered the conjugate point and the Darboux point were determined and the global optimal trajectory was assessed. While in a linear quadratic problem a Darboux point on an extremal occurs at the first backward conjugate point, it is shown that the Darboux point and the conjugate point are distinet in the problems considered in the present analysis. The global optimality is always lost before local optimality.

\section{OPTIMAL SWITCHING}

Consider a dynamical system defined by an $n$-vector $x$ subject to the differential constraint

$$
\dot{x}=f(x, u),
$$

where $u$ is an $m$-control vector belonging to a certain control space $U$

$$
u \in U \text {. }
$$

With the usual assumption of continuity for the functions $f_{j}, j=1,2, \cdots, n$, components of the vector $f$, as well as for their partial derivatives with respect to the state vector components $x_{i}, i=1,2, \cdots, n$, the state equations possess a unique solution for a given initial condition $x\left(t_{1}\right)=x^{4}$ with any prescribed function $u(t) \in U$. 


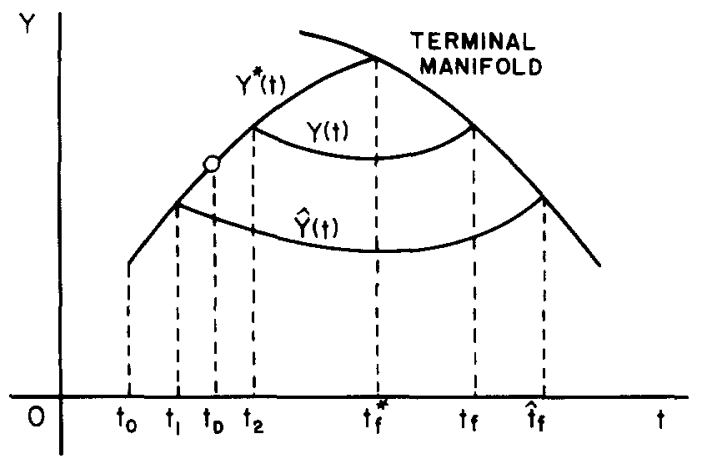

Fig. 1. The Darboux point.

We are concerned with problems in which the initial state is prescribed and the terminal state belongs to a given subset $\theta$ of an $n$-dimensional Euclidean space $R^{n}$. Hence, if $t$, is the final time, $x\left(t_{t}\right) \in \theta$. A control function $u(t)$ is admissible if and only if it is defined and piecewise continuous and $u(t) \in U$ for all $t \in\left[\bar{t}_{1}, \bar{t}_{t}\right]$ with $\left[t_{1,}, t,\right] \subset\left[\bar{t}_{1,}, \bar{t}_{f}\right]$. If in addition, $u(t)$ generates the solution $x(t)$ such that $x\left(t_{t}\right)=x^{i}$ and $x\left(t_{f}\right) \in \theta$, then $u(t)$ is termed feasible at $x^{\prime \prime}$.

The optimal control problem consists of selecting the control $u^{*}(t)$, subject to the constraint (3), such that the initial and final conditions are satisficd and a performance index given by

$$
I=\int_{t}^{t} f_{.}(\mathrm{x} \cdot u) d t
$$

is minimized. The function $f_{\text {" }}$ has the same continuity property as the functions $f$,

We define an augmented state $y=\left(x_{0}, x\right)$ by adding a new state component $x_{\text {, }}$, such that

$$
\dot{x}_{n}=f_{1}(x \cdot u) .
$$

Then in the augmented state space $R^{n \cdot 1}$, the trajectory is governed by the equation

$$
\dot{y}=g(y, u)
$$

where $g=\left(f_{1}, f\right)$. The initial state is $y\left(t_{11}\right)=y^{\prime \prime}=$ $\left(0, x^{6}\right)$.

Following the approach of Contensou[5], we define the natural domain of manueverability $D(y)$ at the time t. with state vector $y$, as the reachable domain in the hodograph space

$$
V=\dot{y}=g(y, u)
$$

Introducing the $(n+1)$-adjoint vector $p$, we obtain the optimal trajectory by selecting at each instant $t$ the control vector $u^{*}$ in the control space $U$ such that

$$
u^{*}=\arg \sup _{u \in \ell^{\prime}} H, \quad \text { or } H^{*}=\sup _{u \in \ell^{\prime}} H
$$

where $H$ is the Hamiltonian

$$
H=p \cdot g=p \cdot V .
$$

In the domain of maneuverability (Fig. 2), the optimal condition (8) leads to the selection of the optimal oper ating point $M^{*}$ such that the projection of the vector $V^{*}=O M^{*}$ on the adjoint vector $P$ is maximized. The point $M^{*}$ is necessarily on the convexized boundary $G$ of $D$. By definition (7), this is equivalent to selecting the optimal control $u^{*}$.

When the convex boundary has a rectilinear part, either natural $R$. or artificial $\bar{R}$ obtained through convexization. a sudden discontinuity from $M_{11} \rightarrow M_{210}$ of the optimal operating point may occur as shown in Fig. 3. We have then a switching. Because of this discontinuity, the optimal trajectory exhibits a corner.

The parameter $t$, termed the time, monotonically in creases during the process. Let $t$, be the switching time We consider the convex parts, $G_{1}$ and $G_{2}$ of the boundary $G$, near the points $M_{111}$ and $M_{20}$. respectively. Let $M_{*}^{*}$ and $M_{2}^{*}$ be the best operating point selected on $G_{1}$ and $G_{2}$. We have the corresponding maximized Hamiltonians

$$
\begin{aligned}
& H_{1}^{*}=\sup _{y+f_{1}} H=H_{1}^{*}(p, y) \\
& H_{2}^{*}=\sup _{y=i_{1}} H=H_{2}^{*}(p, y) .
\end{aligned}
$$

Then, when $t<t$, we select $M_{1}^{*}$, which corresponds to the control $u_{1}^{*}$ and Hamiltonian $H_{1}^{*}>H_{2}^{*}$. When $t>$ $t_{\text {, }}$, we select $M_{2}^{*}$, which corresponds to the control $u^{*}$ and Hamiltonian $H_{2}^{*}>H_{1}^{*}$. At the time $t=t$, we have $H_{1}^{*}=H_{2}^{*}$. Furthermore, we have the switching $M_{4 \mathrm{ti}} \rightarrow$ $M_{211}$. if at $t=1$.

$$
\frac{d}{d t}\left(H_{-}^{*}-H_{i}^{*}\right)>0
$$

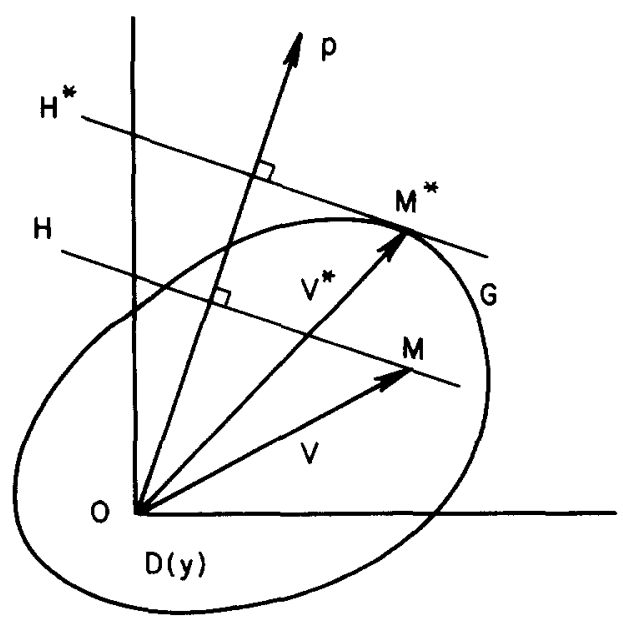

Fig. 2. Selection of the optimal control in the domain of maneuverability. 


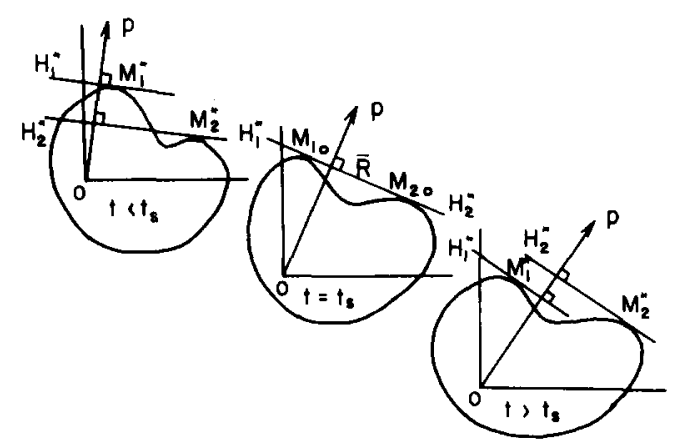

Fig. 3. Optimal switching $M_{11} \rightarrow M_{2,1}$.

since the switching function

$$
\Phi=H_{2}^{*}-H_{i}^{*}=\Phi(p, y)
$$

passes from a negative value to a positive value at that point. We evaluate the time derivative in inequality (11) in the time interval $t \in\left[t_{s}-\epsilon, t_{s}\right]$ that is, with the canonical equations

$$
\frac{d y_{i}}{d t}=\frac{\partial H_{1}^{*}}{\partial p_{i}}, \frac{d p_{i}}{d t}=-\frac{\partial H_{1}^{*}}{\partial y_{i}}
$$

generated by the control $u_{1}^{*}$. Since the system is autonomous, that is $d H_{1}^{*} / d t=\partial H_{1}^{*} / \partial t=0$, we have the necessary condition for the switching $M_{10} \rightarrow M_{20}$

$$
\Omega=\frac{\partial H_{2}^{*}}{\partial y_{i}} \frac{\partial H_{1}^{*}}{\partial p_{i}}-\frac{\partial H_{i}^{*}}{\partial y_{i}} \frac{\partial H_{2}^{*}}{\partial p_{i}} \geq 0
$$

This optimal switching condition, first obtained by Marec in a different form[6], has been extended to the case of a nonautonomous system[7]. It is presented here in a more symmetric form. Similar results for the classical theory of the calculus of variations were obtained by Hefner[8]. In the rare case where we have an equality sign in condition (14), further conditions are required for a switching $M_{10} \rightarrow M_{20}[7]$.

The main result of this section yields the following theorem.

Theorem 1. At each corner of an extremal $y^{*}(t), t \in$ $\left[t_{i}, t_{f}^{*}\right]$, we have the necessary condition

$$
\begin{aligned}
\Omega & =\dot{y}_{i}^{-} H_{y_{i}}^{+}-\dot{y}_{i}^{+} H_{y_{i}} \\
& =g_{i}\left(y, u^{*-}\right) H_{y_{i}}^{+}-g_{i}\left(y, u^{*+}\right) H_{y_{i}} \geq 0 .
\end{aligned}
$$

The condition (15) is simply an explicit form of condition (14) with superscripts $(-)$ and $(+)$ denoting the condition before and after a corner, respectively. We notice that by continuity of the state variables $y_{i}^{-}=$ $y_{i}^{+}$. In the functions $H_{y_{i}}^{-}$and $H_{y_{i}}^{+}$, we substitute the optimal controls $u^{*}$ and $u^{*^{+}}$, respectively, in the Hamiltonian before taking its partial derivative.

\section{CHARACTERISTICS OF A DARBOUX POINT}

From here on, we shall consider a trajectory $y^{*}(t)$ going backward from a point on the terminal manifold $\theta \times R^{\prime}$ to the initial point $y\left(t_{0}\right)=y^{0}$. We assume that this trajectory, which is an extremal, satisfies the maximum principle including the condition in Theorem 1 with the strict inequality sign. The backward approach from the terminal manifold to any current state $y^{*}(\tau)$ implies that $u^{*}(t)$ is feasible at $y^{*}(\tau)$ (also referred to as feasible from $\tau$ ). By optimal control, we mean a control $u^{*}(t)$ that generates a globally optimal trajectory. By the wellknown principle of optimality (see [9], Lemma 10.1), and in view of Definition 1 , if $t_{D}$ is a Darboux point, then $u^{*}(t)$ is an optimal control at $y^{*}(\tau), \tau \in\left(t_{D}, t_{f}^{*}\right)$. It remains to assess the optimality of the trajectory from $t=t_{b}$. For this, we have the following theorem.

Theorem 2. If the problem is normal, and if $t_{D}$ is not a corner for feasible control from $t_{D}$, then the trajectory $y^{*}(t)$ is optimal on $\left[t_{D}, t_{f}^{*}\right]$.

The assumption of normality and the fact that $t_{l}$ is not a corner imply that the initial operating point is selected on the natural convex boundary of the domain of maneuverability, and from any starting point $t=\tau$. the performance index $l[u, \tau]$ is a continuous function of $\tau$. Furthermore, the condition in Theorem 1, which expresses the behavior of the Weierstrass function at a corner of an extremal, insures that whenever there exists a feasible control at any point $A$ of $y^{*}$, there exists a neighboring feasible control starting from any points of $y^{*}$ close enough to $A$. Then, we can follow the same set of arguments as in [4] for the proof of the theorem.

Let $t_{D}$ be a Darboux point on $y^{*}$ and suppose that $y^{*}$ is not optimal on $\left[t_{D}, t_{f}^{*}\right]$. Then, there exists a control $u^{\prime}(t)$, feasible from $t_{D}$ such that

$$
I\left[u^{\prime}, t_{D}\right]<I\left[u^{*}, t_{D}\right]
$$

From the continuity of $I$ with respect to $\tau$, for any given $\eta>0$, there exists $\epsilon_{\eta}>0$ such that

$\left|I\left[u^{*}, t_{D}+\epsilon\right]-I\left[u^{*}, t_{D}\right]\right|<\eta$ for all $\epsilon<\epsilon_{\eta}$.

Also, from the property mentioned above, for any given $\delta>0$, there exists $\epsilon_{\delta}>0$ such that there exists a control $u^{\prime \prime}(t)$, feasible from $t_{D}+\epsilon$ such that

$$
\left|I\left[u^{\prime \prime}, t_{D}+\epsilon\right]-I\left[u^{\prime}, t_{D}\right]\right|<\delta \text { for all } \epsilon<\epsilon_{\delta} .
$$

From the last three conditions, it appears that $\eta$ and $\delta$ can be chosen small enough so that the two balls $\left(I \mid u^{\prime}\right.$, $\left.\left.t_{D}\right], \delta\right)$ and $\left(I\left[u^{*}, t_{D}\right], \eta\right)$ are disjoint and hence, for all $\epsilon<\inf \left(\epsilon_{\eta}, \epsilon_{\delta}\right)$,

$$
I\left[u^{\prime \prime}, t_{D}+\epsilon\right]<I\left[u^{*}, t_{D}+\epsilon\right] .
$$

This is in contradiction with part (i) of Definition 1. Therefore, $y^{*}$ is optimal on $\left[t_{D}, t_{f}^{*}\right]$.

In [4], a Darboux point is characterized as of Type 1 if there exists an optimal trajectory to the problem starting 
from $\left.y^{*}\left(t_{t}\right)-\epsilon\right)$ at $t=t_{1}-\epsilon$ for $\epsilon>0$ arbitrarily small, and as of Type 2 if there does not exist an optimal trajectory to the problem starting from $y^{*}\left(t_{t}-\epsilon\right)$ at $t=t_{t},-\epsilon$ for $\epsilon>0$ arbitrarily small. Then. using the domain of maneuverability, it is clear that for a Type 1 Darboux point, under the assumptions in Theorem 2, at $t=t_{j}$. we have the case of Fig. 4 where there is a possibility of at least another distinet trajectory from $t_{i}$, providing the same minimum cost as the original trajectory.

To justify the condition in Fig. 4. let $u^{*}$ be the feasible control from $t=t_{1},-\epsilon$, which generates the original trajectory $y^{*}$ starting from $y^{*}\left(t_{0},-\epsilon\right)$. We know that this trajectory is optimal on $\left[t_{b}, t_{*}^{*}\right]$. Let $u^{\prime}$ be the optimal control from $t=t_{l}-\epsilon$ which generates the optimal trajectory $y^{\prime}(t)$ starting from $y^{\prime}\left(t_{t}-\epsilon\right)=y^{*}\left(t_{l}-\epsilon\right)$. The existence of this trajectory is from the assumption of Type 1 Darboux point. We wish to prove that $l \mid a^{\prime}$, $t_{p}|=I| u^{*} . t_{l} \mid$ at the limit when $\epsilon$ tends to zero. Suppose that this is not true. that is

$$
|| u^{\prime}, t_{1}|>I| u^{*}, t_{n} \mid .
$$

The domain of maneuverability, and the initial adjoint vector $p$ evolve continuously as the point $\tau$ moves along the trajectory $y^{*}$. This leads to a continuous variation of the performance index $I|u, \tau|$ as a function of $\tau$. Hence. for any given $\eta>0$, there exists $\epsilon_{\eta}>0$ such that

$$
|I| u^{\prime}, t_{D}-\epsilon|-I| u^{\prime}, t_{D}||<\eta \text { for all } \epsilon<\epsilon_{\eta} \text {. }
$$

Also from the property of the existence of a neighboring feasible control, for any given $\delta>0$, there exists $\epsilon_{i}$ 0 such that there exists a control $u^{\prime \prime}$, feasible from $t_{n}$, $\epsilon$ such that

$$
\left.|I| u^{\prime \prime}, t_{b},-\epsilon\right]-I\left|u^{*}, t_{b}\right| \mid<\delta \text { for all } \epsilon<\epsilon_{i n} .
$$

From the last three conditions, we can always select $\eta$ and $\delta$ small enough so that the two balls $\left.\left(I \mid u^{\prime}, t_{1}\right) \mid, \eta\right)$ and $\left(I\left|u^{*}, t_{0}\right|, \delta\right)$ are disjoint and hence

$$
\left.I\left[u^{\prime}, t_{b}-\epsilon\right]>I \mid u^{\prime \prime}, t_{i}-\epsilon\right]
$$

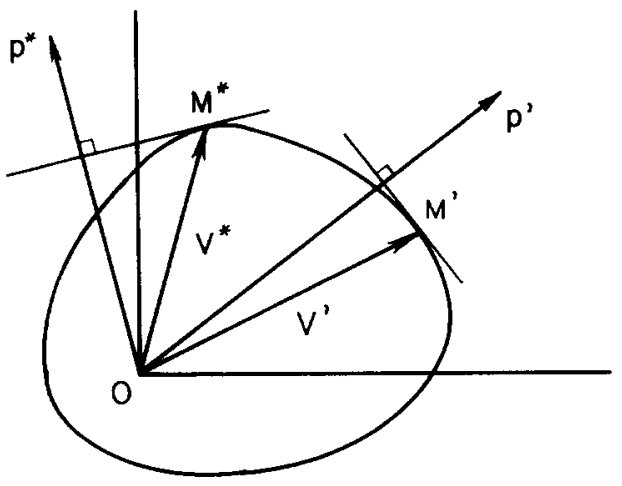

Fig. 4. Domain of maneuverability for Type 1 Darboux point at $t=t s$.

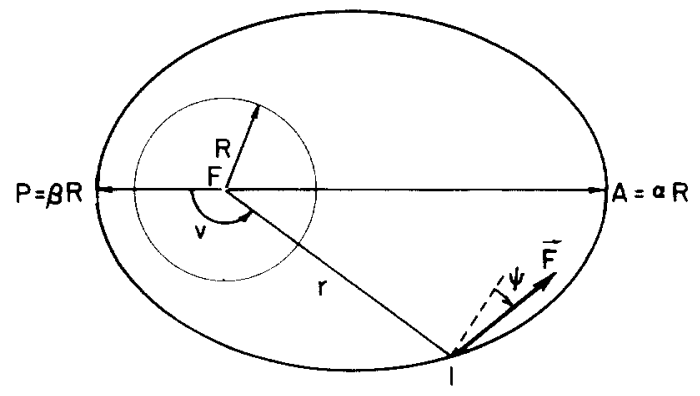

Fig. 5. State and control variables.

This, however, contradicts the assumption that $u^{\prime}$ is the optimal control from $t_{1 \prime}-\epsilon$. Hence $l\left|u^{\prime}, t_{\prime \prime}\right|=I \mid u^{*}$. $t_{i}, 1$.

In general $u^{*}$ and $u^{\prime}$ are distinct as seen in Fig. 4 but there may exist more than one distinct optimal control from $t_{p}$. In the case where $u^{\prime} \equiv u^{*}$, the two vectors $p^{\prime}$ and $p^{*}$ are collinear. Since these are defined by a coef ficient of proportionality which takes into aceount the transversality condition which must be satisfied at the terminal state. the trajectory is unique. Since $u^{\prime}$ tends to $u^{*}$ when $p^{\prime}$ tends to $p^{*}$, the trajectory $y^{\prime}(t)$ starting from $\left.y^{\prime}\left(t_{i},-\epsilon\right)=y^{*}\left(t_{t}\right)-\epsilon\right)$ is in the neighborhood of the trajectory $y^{*}(t)$ starting from $y^{*}\left(t_{l},-\epsilon\right)$ giving a smaller value to the performance index. Hence, there exists a conjugate point $t_{t}$, in the interval $\left(t_{D},-\epsilon, t_{D}\right)$ and at the limit $t_{c}=t_{l}$.

\section{OPTIMIAL PLANAR DEORBIT}

The theory is now applied to find the globally optimal trajectory for the problem of minimum fucl deorbit for a prescribed entry condition.

There is given an elliptical orbit $O_{\text {. about a spherical }}$ planet with center of attraction at $F$. The planet is surrounded by an atmosphere with radius $R$. If the orientation of the line of apsides is free, the orbit is defined by its apocenter distance $A$ and pericenter distance $P$ (Fig. 5). Within the assumption of impulsive change in the velocity upon the application of a thrust, it is proposed to find the minimum fuel deorbit trajectory for entry at distance

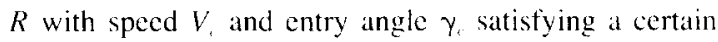
constraint with a general form as given in equ (1). Wo shall consider the case where the entry angle $\gamma$, is pre scribed. Interested readers may apply the theory to any function in the gencral form.

The total characteristic velocity, which is a measure of the fuel consumption for a high thrust propulsion system. is defined by the integral

$$
C=\int_{1,}^{+F} \frac{F}{m} d t \geq 0
$$

where $F$ is the thrust and $m$ is the mass of the vehicle. The control is represented by the vector

$$
n=(1.11)
$$


defining the true anomaly $v$ of the point of application of the thrust and the thrust angle $\psi$ measured from the local horizontal plane. It is convenient to use $R$ as the unit distance and the circular speed at distance $R$ as the unit speed. Hence if $\mu$ is the gravitational constant and a the semimajor axis of the orbit. we define the dimensionless variables

$$
\alpha=\frac{A}{R}, \quad \beta=\frac{P}{R}, \quad c=\frac{C}{\sqrt{\mu / R}}, \quad n=\sqrt{\frac{R^{3}}{a^{3}}} .
$$

From the classical equations of variations in celestial mechanics we have the state equations

$$
\begin{aligned}
\frac{d \alpha}{d c} & =\frac{1}{n} \sqrt{\frac{1+e}{1-e}}\{(1+e) \sin v \sin \psi \\
& +[2+(1+e) \cos v \\
& +(1-e) \cos E] \cos \Phi\} \\
\frac{d \beta}{d c} & =\frac{1}{n} \sqrt{\frac{1-e}{1+e}}\{-(1-e) \sin v \sin \psi \\
& +(2-(1-e) \cos v \\
& -(1+e) \cos E] \cos \psi\}
\end{aligned}
$$

where $e$ is the eccentricity and $E$ is the eccentric anomaly. Introducing the adjoint variables $p_{a}, p_{\beta}$, and $p_{i}$, we form the Hamiltonian

$$
H=p_{\alpha} \frac{d \alpha}{d c}+p_{\beta} \frac{d \beta}{d c}+p_{c}=\Lambda \cdot V+p_{c}
$$

where $\Lambda=\left(p_{c}, p_{\beta}\right)$ and $V$ is the state velocity with components given by the right-hand side of eqns (25).

\section{1 Optimal control}

In the hodograph space $V=\left(\alpha^{\prime}, \beta^{\prime}\right)$, we consider Contensou's domain of maneuverability, which is the reachable domain for all possible values of the control $v$ (and $E$ ) and $\psi$. Let

$$
k_{1}=\frac{1}{n} \sqrt{\frac{1+e}{1-e}}, \quad k_{2}=\frac{1}{n} \sqrt{\frac{1-e}{1+e}} .
$$

When $v=E=0, \beta^{\prime}=0$ and we have the segment $P_{1} P_{2}$ on the $\alpha^{\prime}$ axis between $-4 k_{1}$ and $4 k_{1}$. On the other hand, when $v=E=\pi, \alpha^{\prime}=0$ and we have the segment $A_{1} A_{2}$, on the $\beta^{\prime}$ axis between $-4 k_{2}$ and $4 k_{2}$ (Fig. 6). For constant and arbitrary $v$ between 0 and $\pi$, that is for a fixed position in the orbit with all possible thrust angles, the domain of maneuverability is the ellipse with center at 0 as given by the equation

$$
\begin{aligned}
\left(F_{2} \alpha^{\prime}-F_{2} \beta^{\prime}\right)^{2}+ & \left(G_{2} \alpha^{\prime}-G_{1} \beta^{\prime}\right)^{2} \\
& =16 k_{1}^{\prime} k_{2}^{2} \sin ^{2} v(1-e \cos E)^{2}
\end{aligned}
$$

where

$$
\begin{aligned}
F_{1}= & k_{1} f_{1}=k_{1}(1+e) \sin v \\
G_{1}= & k_{1} g_{1}=k_{1}[2+(1+e) \cos v \\
& +(1-e) \cos E] \\
F_{2}= & k_{2} f_{2}=-k_{2}(1-e) \sin v \\
G_{2}= & k_{2} g_{2}=k_{2}[2-(1-e) \cos v \\
& -(1+e) \cos E] .
\end{aligned}
$$

The ellipse is completely inside of the rhombus $A_{1} P_{1} A_{2} P_{2}$.
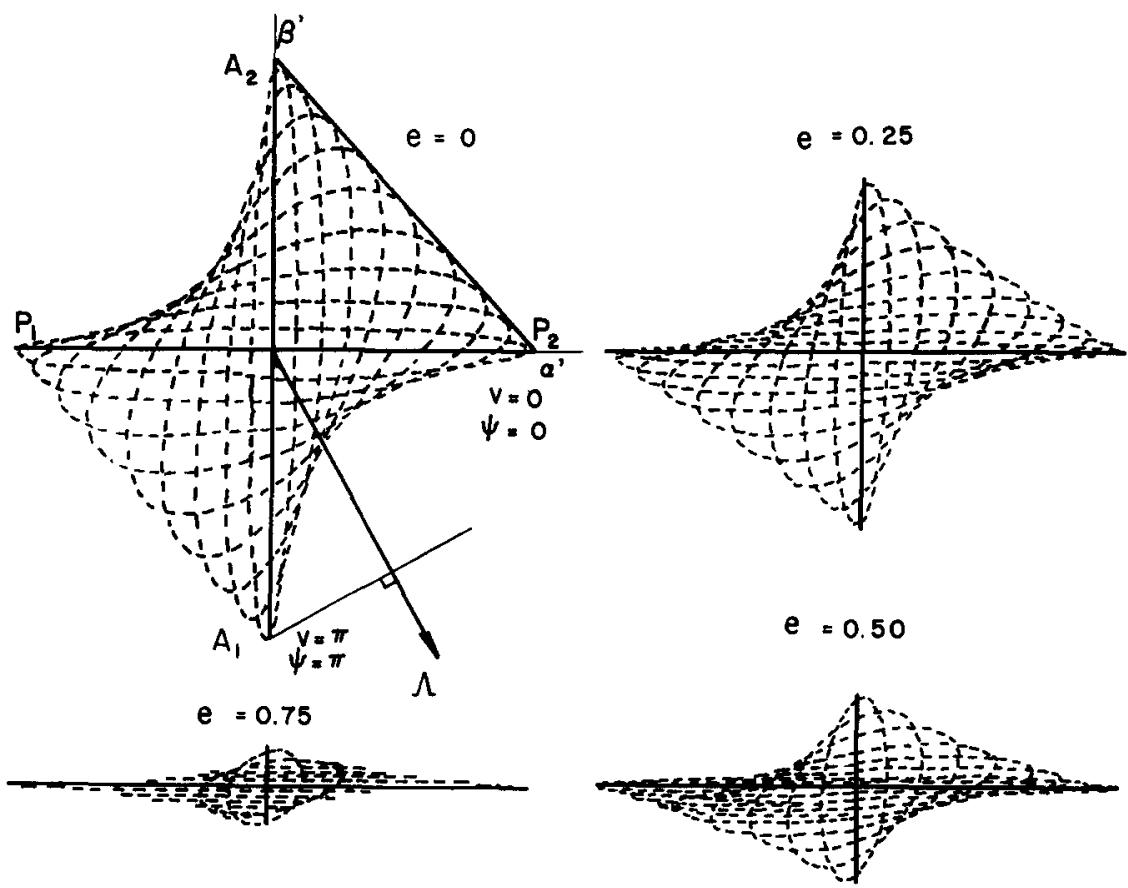

Fig. 6. Domain of maneuverability for various values of the eccentricity $e$. 
To prove this, consider for example the line $A_{2} P_{2}$ with equation

$$
\frac{\alpha^{\prime}}{k_{1}}+\frac{\beta^{\prime}}{k_{2}}=4
$$

Let $\Delta$ be the function on the left-hand side of eqn (28). It suffices to show that $\Delta\left(\alpha^{\prime}, \beta^{\prime}\right)>16 k k_{z}^{2} \sin ^{2} v(1-$ $e \cos E)^{2}$ for all points on the line $A_{2} P_{2}$. Using eqn (30) to express $\Delta$ in terms of $\alpha^{\prime}$ allone, we have

$$
\begin{aligned}
\Delta=k_{2}^{2}\left[\left(f_{1}+f_{2}\right) \alpha^{\prime}-\right. & 4 k_{1} f_{1} l^{2} \\
& \left.+k_{2}^{2} \mid\left(g_{1}+g_{2}\right) \alpha^{\prime}-4 k_{1} g_{1}\right]^{2} .
\end{aligned}
$$

We consider the minimum of $\Delta$. which is attained when

$$
\begin{aligned}
\left(f_{1}+f_{2}\right) \|\left(f_{1}\right. & \left.\left.+f_{2}\right) \alpha^{\prime}-4 k_{1} f_{1}\right] \\
& +\left(g_{1}+g_{2}\right)\left[\left(g_{1}+g_{2}\right) \alpha^{\prime}-4 k_{1} g_{1}\right\rfloor=0 .
\end{aligned}
$$

Then, after some arrangement. we have the minimum value

$$
\Delta^{*}=\frac{\left[16 k_{1} k_{2} \sin v(1-e \cos E)\right]^{2}}{\left(f_{1}+f_{2}\right)^{2}+\left(g_{1}+g_{2}\right)^{2}} .
$$

Hence, the condition for the line $A_{2} P_{2}$ to be completely outside the ellipse for constant arbitrary $r$ is

$$
\left(f_{1}+f_{2}\right)^{2}+\left(g_{1}+g_{2}\right)^{2}<16
$$

Considering the definition of $f$ and $g$, in eqn (29), we have the condition

$$
-\frac{4 e^{2}(3-e \cos E) \sin ^{2} E}{(1-e \cos E)} \leq 0
$$

which is always satisficd.

When $y$ varies. the ellipse sweeps the domain of maneuverability (as shown in Fig. 6 for various values of e) with 4 angular points at the vertices of the rhombus.

For any vector $\Lambda$, the maximization of the Hamiltonian, in the form of the product (26), leads to using one of these points. The optimal impulse is either at the perigee, $v=0$, or at the apogec, $v=\pi$, with tangential thrust, either forward. $\psi=0$. or backward. $\psi=\pi$.

\subsection{The characteristic cures}

In the $(\alpha, \beta)$ space as shown in Fig. 7. with $\alpha \leq \beta$. the optimal trajectory consists of subares parallel to the axes. In this space, the terminal state $\theta$. which consists of all orbits intersecting the atmosphere at the prescribed angle $\gamma_{1}$, is the hyperbola

$$
\theta(\alpha, \beta)=\beta-\frac{(\alpha-1) \cos ^{2} \gamma_{c}}{\left(\alpha-\cos ^{2} \gamma_{c}\right)}=0
$$

Going backward from any point on the final state, we investigate the possibility of a corner at the switching point $S$. First, at the terminal point we have the trans- versality condition expressing the fact that the adjoint vector is orthogonal to the final state, that is

$$
p_{u} \frac{\partial \theta}{\partial \beta}=p_{\beta} \frac{\partial \theta}{\partial \alpha} .
$$

Since the problem is normal. instead of integrating the adjoint equations to obtain $p_{t}$ and $p_{\beta}$ we simply use the key equation of dynamic programming $|9|$

$$
p_{1}=-\frac{i t}{d x}
$$

where $c$ is the optimal cost which can be readily computed from any point on the final are as

$$
c=\sqrt{\frac{2 \beta}{\alpha(\alpha+\beta)}}-\sqrt{\frac{2(\alpha-1)}{\alpha\left(\alpha^{2}-\cos ^{2} \gamma_{1}\right)}} \cos \gamma_{1} .
$$

Then from eqn (34), we have

$$
\begin{aligned}
p_{r .}=(2 \alpha+\beta) & \sqrt{\frac{\beta}{2 \alpha^{3}(\alpha+\beta)^{3}}} \\
& -\frac{\left(2 \alpha^{3}-3 \alpha^{2}+\cos ^{2} \gamma_{r}\right) \cos \gamma}{\sqrt{2(\alpha-1) \alpha^{3}\left(\alpha^{2}-\cos ^{2} \gamma\right)^{2}}}
\end{aligned}
$$

$p_{\beta}=-\sqrt{\frac{\alpha}{2 \beta(\alpha+\beta)^{2}}}$.

We verify that, at the terminal point with eqn (32), the transversality condition (33) is identically satisfied. Hence, eqns (36) give the variation of the adjoint vector along the final arc with constant $\alpha$.

We have seen that the optimal control is

$$
\begin{aligned}
& \sin v=\sin E=0, \\
& \cos v=\cos E=\epsilon_{1}= \pm 1 \\
& \cos \psi=\epsilon_{2}= \pm 1 .
\end{aligned}
$$

With the relations

$$
\frac{\alpha}{\beta}=\frac{1+e}{1-e}, \quad n=\sqrt{\frac{R^{3}}{a^{3}}}=\sqrt{\frac{8}{(\alpha+\beta)^{3}}}
$$

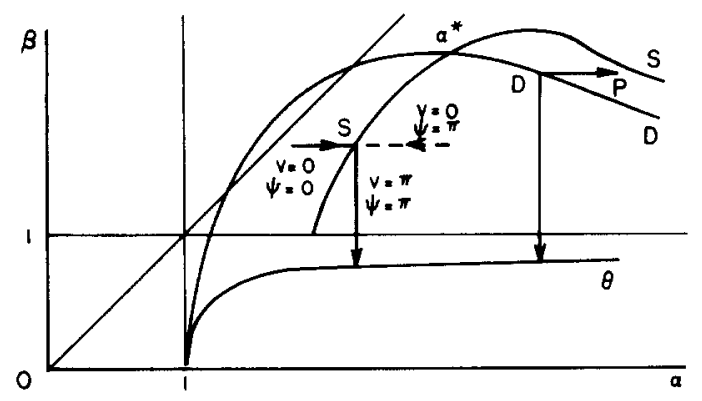

Fig. 7. Final state and optimal switching direction. Possible Darboux point. 


$$
\begin{aligned}
H=\epsilon_{2} \sqrt{\frac{(\alpha+\beta)^{3}}{2 \alpha \beta}}[(1 & \left.+\epsilon_{1}\right) \alpha p_{\alpha} \\
& \left.+\left(1-\epsilon_{1}\right) \beta p_{\beta}\right]+p_{c} .
\end{aligned}
$$

From Fig. 7, we see that after the corner $S$, we have $\boldsymbol{\epsilon}_{1}=-1, \epsilon_{2}=-1$, and hence

$$
H_{2}^{*}=-\sqrt{\frac{2 \beta(\alpha+\beta)^{3}}{\alpha}} p_{\beta}+p_{\imath} .
$$

On the other hand, before the corner, we have the possible arc in solid line with $\epsilon_{1}=1, \epsilon_{2}=1$, and hence

$$
H_{1}^{*}=\sqrt{\frac{2 \alpha(\alpha+\beta)^{3}}{\beta}} p_{\alpha}+p_{c^{\prime}}
$$

If we take the alternate arc, in dashed line, we have $\epsilon_{1}=1, \epsilon_{2}=-1$, and hence

$$
H_{1}=-\sqrt{\frac{2 \alpha(\alpha+\beta)^{3}}{\beta}} p_{\alpha}+p_{c}
$$

The condition (14) with the strict inequality sign, in the present case is reduced to

$$
\Omega=\frac{\partial H_{2}^{*}}{\partial \alpha} \frac{\partial H_{1}^{*}}{\partial p_{\alpha}}-\frac{\partial H_{1}^{*}}{\partial \beta} \frac{\partial H_{2}^{*}}{\partial p_{\beta}}>0 .
$$

At a corner, we have $H_{2}^{*}=H_{1}^{*}$ and since the adjoint vector is continuous across a corner, from eqns (40) and (41), we have a relation at the point $S$

$$
\alpha p_{\alpha}=-\beta p_{\beta}
$$

Again, using eqns (40) and (41) to evaluate the partial derivatives in condition (43) and with the aid of eqn (44) and the second equation (36), we obtain

$$
\Omega=\sqrt{\frac{(\alpha+\beta)^{3}}{2 \alpha \beta}}>0 .
$$

Hence, the condition for optimal switching in Theorem 1 is satisfied. On the other hand, if we use the Hamiltonian $H_{1}$, as given in eqn (42), we obtain through similar calculation

$$
\Omega=-3(\alpha-\beta) \sqrt{\frac{(\alpha+\beta)}{2 \alpha \beta}}<0 .
$$

The dashed arc before the corner is nonoptimal.

Then, using the explicit expressions (36) for the adjoint variables in eqn (44), we have the equation for the curve

$$
\begin{aligned}
& \sqrt{\frac{\beta(\alpha-1)\left(\alpha^{2}-\cos ^{2} \gamma_{e}\right)^{3}}{(\alpha+\beta) \cos ^{2} \gamma_{e}}} \\
& -2 \alpha^{2}(\alpha-1)-\left(\alpha^{2}-\cos ^{2} \gamma_{c}\right)
\end{aligned}
$$

We now consider the possible existence of a Darboux point $D$ along the optimal trajectory, going backward from the final state. This is obtained by writing the condition that at the point $D$ there exists an alternate distinct control providing the same cost. This is the trajectory $D P$ obtained by using $\epsilon_{1}=1, \epsilon_{2}=1$, that is, applying an accelerative impulse at the perigee of the orbit. In the absence of a corner, this trajectory becomes parabolic with a cost

$$
c_{p}=\sqrt{\frac{2}{\beta}}-\sqrt{\frac{2 \alpha}{\beta(\alpha+\beta)}} .
$$

By comparing this cost with the cost given in eqn (35), we have the equation for the curve $D$

$$
\beta=\frac{4(\alpha-1)\left(\alpha^{2}-\cos ^{2} \gamma_{e}\right) \cos ^{2} \gamma_{t}}{\alpha\left(\alpha-\cos ^{2} \gamma_{e}\right)^{2}} .
$$

Whether or not a point on this curve is a Darboux point depends on its relative position with respect to the curve $S$ as will be shown in the subsequent analysis.

Finally, using hodograph theory, at constant $\alpha$, it is easy to show that a trajectory going backward from the terminal state loses relative minimum at a point $C$, the conjugate point, such that

$$
\sqrt{\frac{\beta}{\alpha+\beta}}=\frac{\alpha^{2}}{\cos \gamma} \sqrt{\frac{(\alpha-1)}{\alpha^{2}-\cos ^{2} \gamma}}
$$

The plot of this equation in the $(\alpha, \beta)$ space is referred to as the curve $C$.

\subsection{Optimal trajectories}

For a given initial orbit defined by the point $\left(\alpha_{11}, \beta_{11}\right)$ in the phase space, and a prescribed entry angle $\gamma_{c}$, the optimal trajectory is one of three types. We have the oneimpulse deorbit (I) obtained by applying a tangential and decelerative impulse at the apogee. The second type is a two-impulse deorbit (II) achieved by first applying a tangential and accelerative impulse at the perigee of the initial orbit to raise the apogee from $\alpha_{1}$ to a higher value $\alpha$. This value is obtained by solving eqn (47) for $\alpha$ with $\beta=\beta_{o}$. A second and decelerative impulse is next applied at this new apogee to complete the transfer. A third type is the parabolic deorbit $(P)$. For this type a tangential and accelerative impulse is applied at the perigee of the initial orbit to send the vehicle into a parabolic orbit. At infinity, or in practice at a large distance, an infinitesimal impulse is applied to return the vehicle for reentry at any prescribed angle. The type of optimal trajectory depends on the position of the given point $\left(\alpha_{i}, \boldsymbol{\beta}_{i}\right)$ with respect 
to the curves $D$ and $S$. It is easy to see that a point on the curve $D$ is a Darboux point if and only if the backward trajectory intersects it first. On the other hand, a point on the curve $S$ is a corner if and only if the backward trajectory intersects it first. A complete knowledge of the behavior of the curves $D$ and $S$ as the entry angle $\gamma_{t}$ varies allows a synthesis of the problem.

First. the two curves have the same asymptote

$$
\beta=4 \cos ^{2} \gamma
$$

as $\alpha \rightarrow x$. This curve is referred to as the curve $A$.

Next, the two curves intersect each other at a point (*) where the curve $D$ passes through a maximum. The abcissa of this point is obtained from the equation

$$
\begin{aligned}
\left(1-2 \cos ^{2} \gamma_{1}\right) \alpha^{3} & +3 \alpha^{2} \cos ^{2} \gamma \\
& -3 \alpha \cos ^{2} \gamma_{i}+\cos ^{4} \gamma_{r}=0
\end{aligned}
$$

while the corresponding value of $\beta$ is either given by eqn (49), if $\alpha$ has been computed, or obtained by solving directly the equation

$$
\cos ^{2} \gamma=\frac{27 \beta^{2}}{(4+\beta)^{2}}
$$

If $\beta^{*}$ is the value obtained from this equation, the curve $T$ which is the tangent to the curve $D$ at the point (*) has the equation

$$
\beta=\beta^{*}
$$

Depending on the prescribed value of $\gamma_{1}$, there are three cases as shown in Fig. 8.
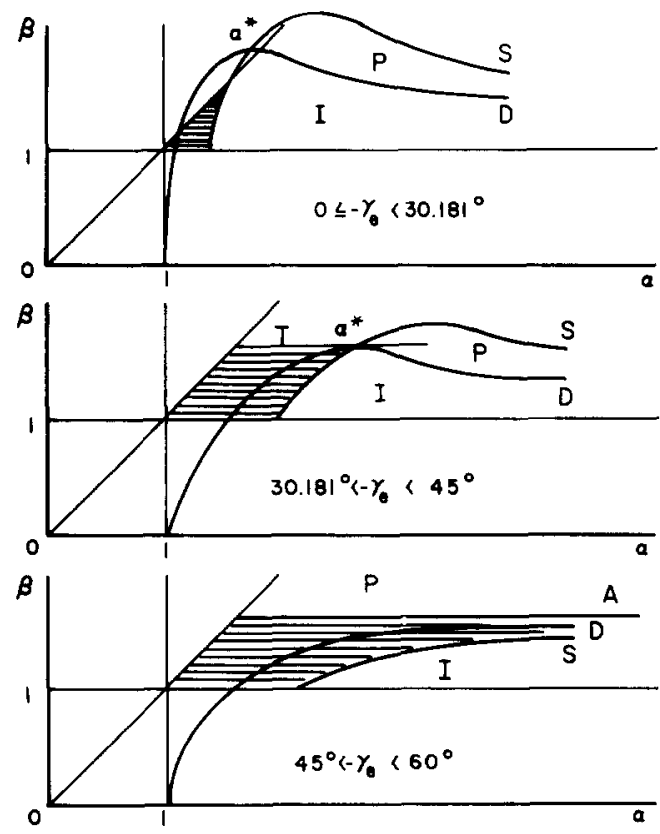

Fig. 8. Behaviour of the characteristic curves. Region (II) in hatched area
For $-\gamma$, between the values $0^{\circ}$ and $30.181^{\circ}=\arccos$ $[137 \sqrt{2}-193]^{1: 2}$ the point $(*)$ is above the bisector $\beta=\alpha$. Let subscript $A$ denote the space above any curve considered and subscript $B$ for the space below that curve. Let $E$ be the space of all elliptic orbits $\alpha \geq \beta$. By simple logic, we conclude for this case the following optimal spaces

$$
\begin{aligned}
& 1 \subset D_{B} \cap S_{B} \\
& \mathrm{I} \subset S_{1}\left(\alpha \leq \alpha^{*}\right) \\
& P \subset D_{3}\left(\alpha>\left(\alpha^{*}\right) .\right.
\end{aligned}
$$

When $-\gamma=30.181^{\circ}$. we have $\alpha^{*}=\beta^{*}=$ $3 \sqrt{2}-1$. For $-\gamma$, between $30.181^{\circ}$ and $45^{\circ}$, the point $(*)$ is in the space $E$ with the curve $S$ tending to the asymptote above the curve $D$. For this case, we have the following optimal spaces

$$
\begin{aligned}
& I \subset D_{k} \cap S_{B} \\
& \text { II } \subset T_{B} \cap S_{1}\left(\alpha \leq \alpha^{*}\right) \\
& P \subset T_{H} \cup D_{*}\left(\alpha \geq \alpha^{*}\right) .
\end{aligned}
$$

When $-\gamma=45^{\circ}$ the point $(*)$ is at infinity. For $-\gamma$ between $45^{\circ}$ and $60^{\circ}$ the two curves $D$ and $S$ have no intersecting point in the space $E$ and they tend to the common asymptote with the curve $D$ above the curve $S$. For this case. we have the following optimal spaces:

$$
\begin{aligned}
& \mathrm{I} \subset S_{k} \\
& \mathrm{II} \subset S_{A} \cap A_{k} \\
& \mathrm{P} \subset A_{k} .
\end{aligned}
$$

When $-\gamma_{r}=60^{\circ}$, the asymptote is $\beta=1$. For $-\gamma_{c}>$ $60^{\circ}$. only the parabolic mode is optimal. It can be easily shown that along the backward trajectory the Darboux point is met before the conjugate point.

Based on this analysis, we have computed the different regions of optimality in the $\left(\beta_{,},-\gamma_{c}\right)$ space using the eccentricity $e_{i}$, fhe initial orbit as a parameter (Fig. 9)

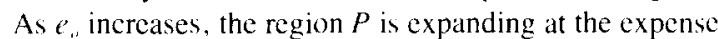
of the regions I and II. But at the same time the region I is expanded into the region II. When $e_{1} \rightarrow 1$, the region II disappears and physically the one-impulse mode is identical to the parabolic mode. Nevertheless. mathematically we have a limiting boundary between the two modes and it is given explicitly by eqn (51) with $\beta=\beta_{n}$.

\section{OPTIMAL NONCOPLANAR DEORBIT}

As a second example, we consider the problem of minimum fuel deorbit for entry at a prescribed angle $\gamma$ with the added condition that a plane change at a prescribed value $i_{f}$ is performed during the maneuver.

The same approach can be used for this case and because of the restriction in the length of the paper, we 


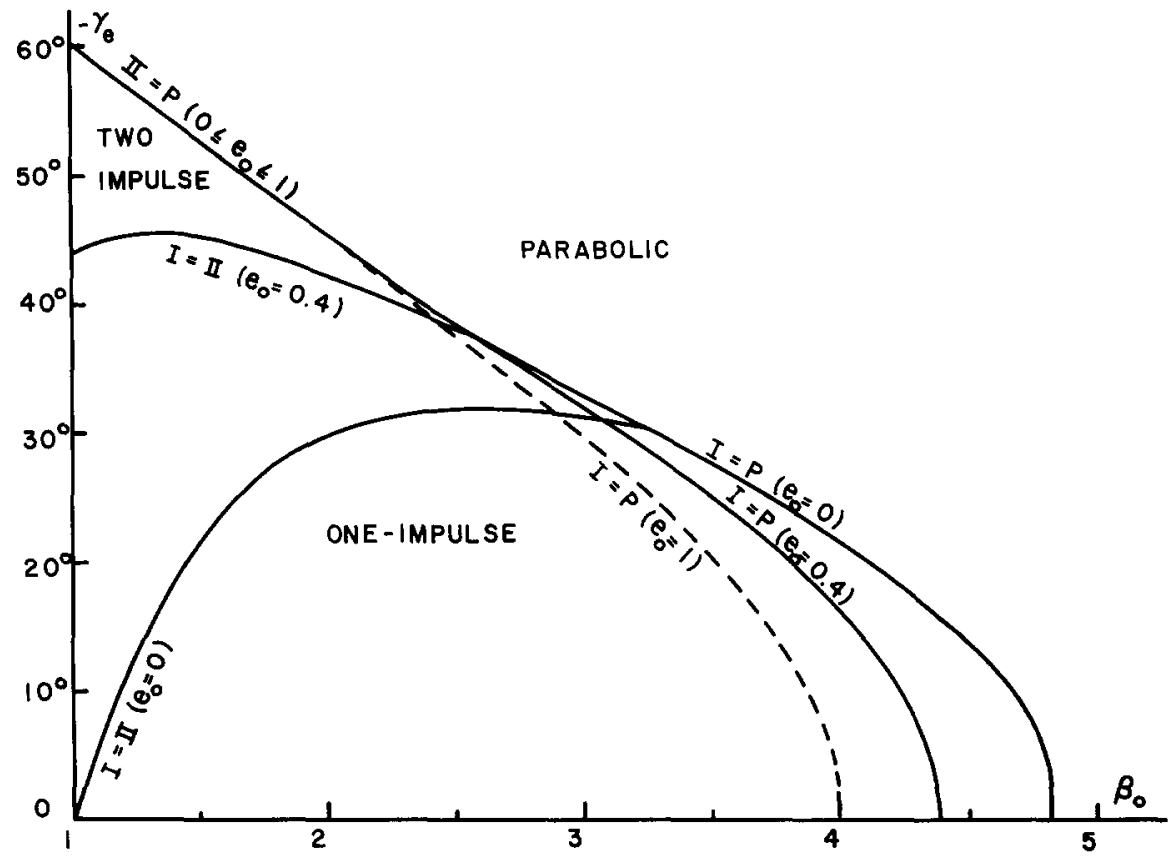

Fig. 9. Regions of optimality for deorbit at prescribed entry angle.

shall give below the main results with all the pertinent equations for the computation of the optimal trajectory.

If the longitude of the ascending node is removed from consideration, we can consider the subspace $(\alpha, \beta, i)$ where $i$ is the inclination measured from the plane of the initial orbit. We have the state equations[10]

$$
\begin{aligned}
\frac{d \alpha}{d c} & =\frac{1}{n} \sqrt{\frac{1+e}{1-e}}\{(1+e) S \sin v \\
& +(2+(1+e) \cos v+(1-e) \cos E] T\} \\
\frac{d \beta}{d c} & =\frac{1}{n} \sqrt{\frac{1-e}{1+e}}\{-(1-e) S \sin v \\
& +[2-(1-e) \cos v-(1+e) \cos E] T\} \\
\frac{d i}{d c} & =\frac{(1-e \cos E)}{n \sqrt{\alpha \beta}} W \cos u
\end{aligned}
$$

where $u=\omega+v$, with $\omega$ being the argument of the perigee and $S, T, W$ are the direction cosines of the impulses with respect to the radial, normal and binormal axes from the plane of the osculating orbit.

As before, the location of the impulses is at the apses, with $\sin v=0, \cos v=\cos E=\epsilon_{1}$, with horizontal application, $S=0$. It turns out that we always have $\cos$ $u=\epsilon_{1}$ if we choose the angle $i$ to be positive, counterclockwise as viewed from the perigee and such that dil $d c>0$. Let $\phi$ be the thrust angle measured from the osculating plane. Then

$$
T=\cos \phi, \quad W=\sin \phi .
$$

With this, we introduce the additional adjoint component $p_{i}$ to write the Hamiltonian

$$
\begin{aligned}
H & =\sqrt{\frac{(\alpha+\beta)^{3}}{2 \alpha \beta}}\left\{\left[\left(1+\epsilon_{1}\right) \alpha p_{u}\right.\right. \\
& \left.+\left(1-\epsilon_{1}\right) \beta p_{\beta}\right] \cos \phi \\
& \left.+\frac{\left(\epsilon_{1}-e\right)}{2} p_{i} \sin \phi\right\}+p_{c} .
\end{aligned}
$$

The terminal state $\theta$, in the cylindrical coordinates space $(\alpha, \beta, i)$, is now the intersection of the hyperboloid as given by eqn (32), and the plane

$$
i-i_{1}=0 \text {. }
$$

At each application of the impulse we have a plane change. The optimal trajectory is either by one impulse at the apogee, or by two impulses with the first impulse at the perigee and the second impulse at the new apogee, or via parabolic orbit. The cost for parabolic transfer is given in eqn (48) and it is independent of $\gamma_{r}$ and $i_{j}$. Going backward from a point on the final state, with constant $\alpha$, the cost from any point along the trajectory is given by

$$
\begin{aligned}
& c=\left[\frac{2 \beta}{\alpha(\alpha+\beta)}+\frac{2(\alpha-1) \cos ^{2} \gamma_{i}}{\alpha\left(\alpha^{2}-\cos ^{2} \gamma_{e}\right)}\right. \\
& \left.-\frac{4}{\alpha} \sqrt{\frac{\beta(\alpha-1) \cos ^{2} \gamma_{i}}{(\alpha+\beta)\left(\alpha^{2}-\cos ^{2} \gamma_{i}\right)}} \cos \left(i_{f}-i\right)\right]^{1.2}
\end{aligned}
$$

The cost for the one impulse deorbit is obtained by putting $i=0$ in this equation. Setting it equal to the parabolic 
cost, we have the equation for the curve $D$ in the plane $i=0$.

It remains to compute the cost for the two-impulse mode, or what is the same, to obtain the equation for the switching surface $S$ and the optimal directions of the impulses.

Before the corner $S$, with the impulse applied at the perigee we have $\epsilon_{1}=1$ with constant $\beta=\beta_{i,}$. Then

$$
\begin{aligned}
& H_{1}=\sqrt{\frac{(\alpha+\beta)^{3}}{2 \alpha \beta}}\left[2 \alpha p_{\alpha} \cos \phi\right. \\
&\left.+\frac{\beta}{\alpha+\beta} p_{i} \sin \phi\right]+p_{r} .
\end{aligned}
$$

The Hamiltonian is maximized with respect to $\phi$ when

$$
2 \alpha(\alpha+\beta) p_{i r} \sin \phi=\beta p_{i} \cos \phi
$$

This results in

$$
H_{1}^{*}=\sqrt{\frac{(\alpha+\beta)}{2 \alpha \beta}} \sqrt{4 \alpha^{2}(\alpha+\beta)^{2} P_{a}^{2}+\beta^{2} p_{i}^{2}}+p_{i \cdot}
$$

Similarly, after the corner $S$, with the impulse applied at the new apogee $\alpha$, we have $\epsilon_{1}=-1$ and a relation for the optimal thrust direction

$$
2 \beta(\alpha+\beta) p_{\beta} \sin \phi=-\alpha p_{i} \cos \phi .
$$

The corresponding maximized Hamiltonian is

$$
H_{2}^{*}=\sqrt{\frac{(\alpha+\beta)}{2 \alpha \beta}} \sqrt{4 \beta^{2}(\alpha+\beta)^{2} p_{\beta}^{2}+\alpha^{2} p_{i}^{2}}+p .
$$

Let $\delta_{1}$ be the angle of the first impulse, and $\delta_{2}$ the angle of the second impulse, both measured from the plane of the first transfer orbit as shown in Fig. 10. Then, from eqns (64) and (66), we have at the switching point $S$

$$
\begin{gathered}
2 \alpha\left(\alpha+\beta_{n}\right) p_{c} \sin \delta_{1}=\beta_{v} p_{i} \cos \delta_{1} \\
2 \beta_{o}\left(\alpha+\beta_{n}\right) p_{\beta} \sin \delta_{2}=-\alpha p_{i} \cos \delta_{2} .
\end{gathered}
$$

At this point, we have $H_{!}^{*}=H_{2}^{*}$ and hence

$$
\begin{aligned}
4 \alpha^{2}\left(\alpha+\beta_{0}\right)^{2} p_{i}^{2}+ & \beta_{i i}^{2} p_{i}^{2} \\
& =4 \beta_{i}^{2}\left(\alpha+\beta_{0}\right)^{2} p_{\beta}^{2}+\alpha^{2} p_{i}^{2} .
\end{aligned}
$$

Substituting eqns (68) into this equation, we have the relation between the optimal angles for the impulses

$$
\alpha \sin \delta_{1}+\beta_{,} \sin \delta_{2}=0 \text {. }
$$

From the geometry of the impulses we easily deduce the constraining relations

$$
\begin{aligned}
\frac{\sin \left(i_{1}+\delta_{1}\right)}{\sin \delta_{1}} & =\sqrt{\frac{\alpha\left(\alpha_{0}+\beta_{0}\right)}{\alpha_{1 \prime}\left(\alpha+\beta_{0}\right)}} \\
\frac{\sin \left(i_{2}+\delta_{2}\right)}{\sin \delta_{2}} & =\sqrt{\frac{\beta_{0}\left(\alpha^{2}-\cos ^{2} \gamma_{c}\right)}{(\alpha-1)\left(\alpha+\beta_{n}\right) \cos ^{2} \gamma}} \\
i_{1}+i_{2} & =i_{f}
\end{aligned}
$$

with the magnitude of the impulses

$$
\begin{aligned}
c_{1}=\frac{\sin i_{1}}{\sin \delta_{1}} \sqrt{\frac{2 \alpha_{0}}{\beta_{0}\left(\alpha_{1}+\beta_{i \prime}\right)}}, \\
c_{2}=-\frac{\sin i_{2}}{\sin \delta_{2}} \sqrt{\frac{2(\alpha-1) \cos ^{2} \gamma_{i}}{\alpha\left(\alpha^{2}-\cos ^{2} \gamma_{1}\right)}} .
\end{aligned}
$$

In these equations, $\alpha_{i}, \beta_{i}$ are the elements of the given initial orbits and $i_{1}$ and $i_{2}$ are the plane change angles upon the applications of the impulses. Of course, $\alpha$ denotes the unknown apogee distance of the intermediary orbit. The five unknowns $\alpha, i_{1}, i_{2}, \delta_{1}$ and $\delta_{2}$ are deter mined by the four equations (70) and (71) and the switching relation to be derived. For this purpose, we utilize eqn (34) with the optimal cost as given by eqn (62) to derive the adjoint components. If it is done and the resulting expressions are evaluated at the switching point $S$ in going backward, that is with $\beta=\beta_{1,}, i_{1}-i=$ $i_{1}-i_{1}=i_{2}$, we have in particular for $p_{\mathrm{s}}$ and $p$

$$
\begin{aligned}
& p_{\alpha}=\frac{1}{c_{2}}\left\{\frac{\beta_{o}\left(2 \alpha+\beta_{o}\right)}{\alpha^{2}\left(\alpha+\beta_{n}\right)^{2}}\right. \\
& +\frac{\left(2 \alpha^{3}-3 \alpha^{2}+\cos ^{2} \gamma_{c}\right) \cos ^{2} \gamma^{2}}{\alpha^{2}\left(\alpha^{2}-\cos ^{2} \gamma_{c}\right)^{2}} \\
& -\frac{\beta_{0} \cos i_{2}}{\alpha^{2}\left(\alpha+\beta_{n}\right)} \\
& \times \sqrt{\frac{\left(\alpha+\beta_{i}\right) \cos ^{2} \gamma_{r}}{\beta_{c}(\alpha-1)\left(\alpha^{2}-\cos ^{2} \gamma_{i}\right)}} \\
& \times\left[\frac{(\alpha-1)\left(2 \alpha+\beta_{i \prime}\right)}{\left(\alpha+\beta_{i \prime}\right)}\right. \\
& \left.\left.+\frac{\left(2 \alpha^{3}-3 \alpha^{2}+\cos ^{2} \gamma_{e}\right)}{\left(\alpha^{2}-\cos ^{2} \gamma_{0}\right)}\right]\right\} \\
& p_{i}=\frac{2 \beta_{o}(\alpha-1) \sin i_{7}}{\alpha\left(\alpha+\beta_{c}\right) c_{2}} \\
& \times \sqrt{\frac{\left(\alpha+\beta_{u}\right) \cos ^{2} \gamma}{\beta_{i}(\alpha-1)\left(\alpha^{2}-\cos ^{2} \gamma\right)}} .
\end{aligned}
$$

Using these expressions in the first of the equations (68), with the aid of eqn (70) and the second of the equations (71), we obtain after some arrangement the required 


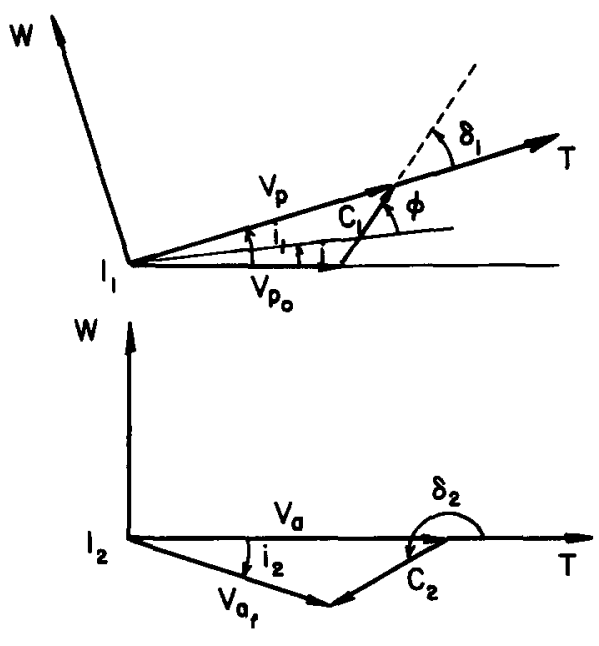

Fig. 10. Geometry of the impulses.

switching relation

$$
\begin{gathered}
\frac{\left(2 \alpha^{3}-3 \alpha^{2}+\cos ^{2} \gamma_{e}\right) \sin i_{2}}{\alpha\left(\alpha^{2}-\cos ^{2} \gamma_{c}\right) \sin \delta_{2}} \\
\times \sqrt{\frac{\left(\alpha+\beta_{o}\right) \cos ^{2} \gamma_{c}}{\beta_{o}(\alpha-1)\left(\alpha^{2}-\cos ^{2} \gamma_{c}\right)}} \\
+\frac{\left(\cos \delta_{1}+\cos \delta_{2}\right)}{\left(\alpha+\beta_{i}\right)} \\
-\frac{\left(\alpha^{2}-2 \alpha+\cos ^{2} \gamma_{c}\right) \cos \delta_{2}}{(\alpha-1)\left(\alpha^{2}-\cos ^{2} \gamma_{e}\right)}=0 .
\end{gathered}
$$

of maxima and minima to minimize the total $\cos t c=$ $c_{1}+c_{2}$, as given in eqn (72), subject to the constraints (71). The necessary conditions obtained are precisely the two relations (70) and (74).

The variational approach used in this paper is more rigorous and it leads directly to the selection of the globally optimal trajectory. We can verify that the switching perigees $\rightarrow$ apogee is indeed optimal since by using the Hamiltonians (65) and (67) to verify the condition (14), we obtain

$$
\begin{aligned}
\Omega=\frac{\left(\alpha+\beta_{i}\right) p_{i} \sin \left(\delta_{1}+\delta_{2}\right)}{2 \sin \delta_{1}} \sin \delta_{2} \\
\times\left[2+\cos \left(\delta_{1}+\delta_{2}\right)\right]>0 .
\end{aligned}
$$

As a numerical application, we have computed the optimal trajectories for deorbit from a circular orbit with $\alpha_{0}=\beta_{0}=r_{0} / R=n$ for entry at a prescribed angle $\gamma_{t}$ with a total plane change $i_{f}$. This is a problem of practical interest in aeroassisted maneuvers. For a minimum fuel return from a high orbit to a low orbit with plane change using aerodynamic force, a preliminary propulsive maneuver with plane change is always made with a prescribed reentry angle to insure a subsequent successful aerodynamic skip trajectory.

The results are presented in Fig. 11 which plots the different optimal regions in the $\left(n, i_{f}\right)$ space for various entry angles. For clarity of the plot, we have presented the deliminating curves for the two values $\gamma_{t}=0^{\circ}$ and $\gamma_{c}=-10^{\circ}$. Let

$$
X=\left(n^{2}-\cos ^{2} \gamma_{t}\right) / n^{2}
$$

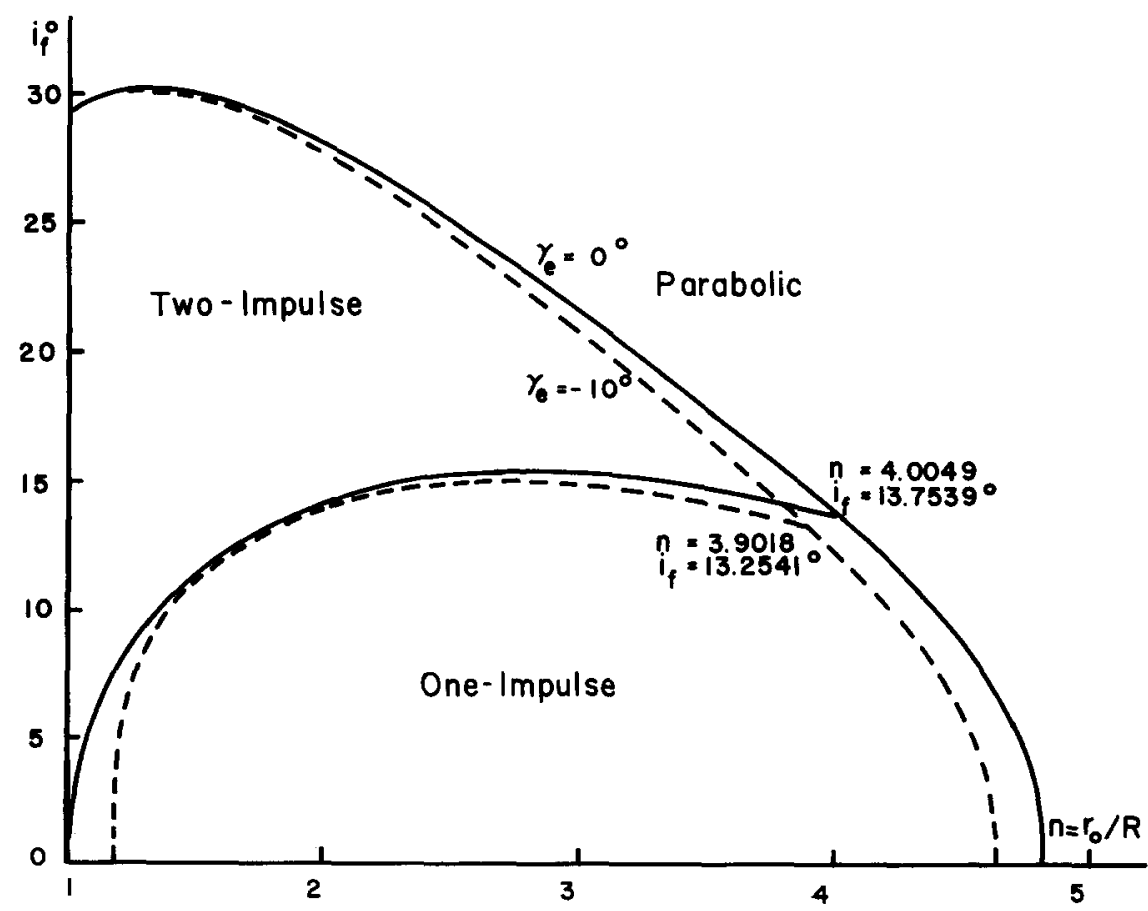

Fig. 11. Regions of optimality for deorbit for prescribed entry angle and plane change. 
It can be shown that the curve between the (I) and the $(P)$ regions is given by the explicit equation

$$
\cos i_{f}=\frac{(n-1)-(n-\sqrt{2}) X}{\sqrt{2(n-1) X(1-X)}}
$$

while the explicit equation for the deliminating curve between the (I) and (II) regions is

$\cos i_{1}$

$$
=\frac{(n-1)\left[3 X^{2}-2(2 n-1) X+4(n-1)\right]}{[2(n-1)+(n-2) X] \sqrt{2(n-1) X(1-X)}} .
$$

Acknowledgement-This work was supported in part by the Jet Propulsion Laboratory under Contract 956416 with Dr. K. D. Mease as project monitor.

\section{REFERENCES}

1. H. G. Moyer and H. J. Kelley. Conjugate points on extremal rocket paths. Proc. 19th IAF Congr. Pergamon Press. ()xford (1970).

2. W. G. Melbourne. The conjugate point and dynamic programming. JPL Rep. No. 32-1043 (1966).

3. G. Darboux, Leçons sur la théorie générale des surfaces. Vol. III, Gauthiers Villars, Paris (1894)

4. P. M. Mereau and W. F. Powers, The Darboux point. JOTA. 17, 545-559 (1975)

5. P. Contensou, Conditions d'optimalité pour les domaines de mannoeuvrabilité à frontière semi-affine. Lecture Notes in Mathematics, No. 112, Springer-Verlag (1970)

6. J. P. Marec. Optimal Space Trajectories. Elsevier, Amsterdam (1979)

7. N. X. Vinh, Optimal Trajectories in Atmospheric Flight Elsevier, Amsterdam (1981)

8. R. A. Hefner. The condition of Mayer for discontinuous solutions of the Lagrange problem. Ductoral thesis. Lniv. Chicago (1931).

9. G. Leitmann. The Calculus of Veriation and Optimal $\mathrm{Con}$ trol. Plenum, New York (1981)

10. C. Marchal. Transferts optimaux entre orbites elliptiquesdurée indifférente. Doctoral thesis, Lniv. Paris (1967). 